Rivera M. y cols.

Rev. Chil. Pediatr. 66 (3); 150-155, 1995

\title{
Evaluación de cuatro métodos para detección de rotavirus en deposiciones en niños chilenos
}

\author{
Maribel Rivera M. ${ }^{1}$; Pablo A. Vial C. ${ }^{3}$ : Marcela Potin S. ${ }^{1}$; \\ Priscilla Prado D. ${ }^{1}$ : Patricia Amarales O. ${ }^{3}$; Miguel O'Ryan G. \\ Marcela Ferrés C. ${ }^{1}$; Katia Abarca $V^{1}$; Francisco Montjel A. ${ }^{2}$
}

Comparisson of four different tests for rapid diagnosis of rotavirus infection in infants

In order to compare different lests for tre rapid diagrosis of rolavirus infection in inionts, fiftyone stool specimens from chilecn chilcren with acute gastrontestinal disease we'e tested for the presence of this virus by gei elevt'ophores's of vircl RivA (Rotagei, ISP-Chilel. latex agglut nation assay i Rotovirus Arit gen 'arex Test iRALTi!. Meridiant. ELISA (Pathfinder, Kaliestud!, and latex-based ElÁ IRVTest Pack, Abbotti. All samples were a'so onalyzed by a olocking ELISA contirmatio- test which was considered os tha gold slondard. Sensirivity/specility volues for all tests were: ELLSA: 100/90\%: TestPack: 95/97\%; Rolagel: 81/100\%; RA_T: 7:/100\%. Processing lime ranged from 15 mir?

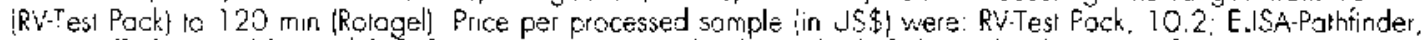
0.7; RALY, 2.4; and kotagel 0.5. RV-Tes Pack seemis to be the method of choice for diagnos's cf roravirus ossociatec dicrihea because it is ieliable and faslest. thougn its highet price may praclude its use in many clinical centers. EllSA shows similar diagrostic efficiency, it is less expensive but reauires a longe' processing t'me and higher rechnical shills.

'Kay words: rotavirus, diognoslic leste.'

El descubrimiento de rotavirus (RV) mediante inmuno-electromicroseopía permitió caracterizar la morfologia del virus $y$, consecuente. mente, utilizar la microscopía electrónica (ME) como método para su detección en deposiciones ${ }^{1-4}$. Los estudios iniciales confirmaron la asociación de RV con gastroenteritis y definieron su comportamiento epidemiológico en la población general ${ }^{4.5}$. La caracterización estructural y genómica del virus condujo al desarrollo de métodos de diagnóstico basados en la identificación de sus antígenos o material genómico en deposiciones ${ }^{6-11}$. La menor complejidad de

1. Departamento de Pediatría, Facultad de Medicina, Universidad Católica de Chile.

2. Laboratorio de Microbiología, Facuhad de Medicina, Universidad Católica de Chile.

3. Hospital Regional de Punta Arenas.

4. Departamento de Microbiología, Facultad de Medicina, Universidad de Chile.

Financiamiento del Proyecto de Child Health Foundation UCIID92. estas técnicas ha permitido disponer universalmente de métodos de diagnóstico de RV, estableciéndose que este agente constituye una de las causas más frecuentes de diarrea aguda en niños en diversas regiones del mundo $0^{8,12-14}$. Las técnicas de identificación de RV actualmente disponibles se basan en alguno de los siguientes mêtodos: observación directa (ME), detección de antígenos (inmunoensayos como ELISA $y$ aglutinación partículas) o detección de ARN (electroforesis en gel, hibridización, amplificación genética). Estas técnicas requieren que el virus este presente en una concentración de $10^{4}$ - $10^{5}$ partículas virales por gramo de deposición en el caso de ME, ELISA, aglutinación de láte $x^{2,3,14 \cdot 15}$ o de $10^{7}$ a $10^{11}$ partículas virales por gramo en el caso de electroforesis de $\mathrm{ARN}^{7.9}$. Se ha establecido que durante la infección aguda el RV es excretado en una concentración de al menos $10^{7}$ partículas virales por gramo de deposición $^{9.15,17}$, lo que teóricamente predice que la electroforesis de ARN podría tener una menor sensibilidad en algunas circunstancias. En el 
caso de los inmunoensayos las variaciones observadas en sensibilidad y especificidad se rclacionan aparentemente con factores tales como manejo de la muestra (transporte. conservación. tiempo entre colección y procesamiento), calj. dad de los anticuerpos de captura, presencia en la muestra dc enzinas que degradan proteinas o ácidos nucleicos. Estos factores hacen aconsejable que se evalúe la eficiencia diagnóstica de los diversos métodos en condiciones semejarites a las circunstancias en que se presentan los pacientes infectados ${ }^{11 .}$ 14. 18-20. En la presente investigación se comparan los resultados obtenidos por cuatro métodos diagnósticos disponibles en Chile, a saber: ELISA, aglutinación de látex. ELISA acoplado a aglutinación de particulas (RV-Test Pack) y electroforesis de ARN (Rotagel $(\mathbb{B})$.

\section{Material y métodos}

Como purte de un estuctio prospectivo de ctrologia de diarrea aguda en población nediátrica se seleccionó al azas una sohorte de pacientes para evaluacion de cualro metodos de diagnístice de rotavirus. Las muestras de deposición fucrón obtenidos al momento del diagnóstico de diarrea aguda y alicuoladas en cuatro tubos estériles. Una de ias alícuotas fue anaizada inmedistamente despues de obtenida por clectroforests de ARN cn el laboratorio del Hospiral Parrocuial de San Beruardo según las indicaciones del fabricante (Rolagel. Instituto de Salud Pública de Chile. Santiago); brevemence, se rcaliza extracción del ARN viral Imediante exposición de la muestra a SDS, fenol $y$ cloroformo; el material obtenido se separa electroloréticamente en un gel de poliacrilannidil y se visualiza mediantc tinción argentica. De este modo esta lécnica permite la identificacion del ARN de $R V$ que se caracteriza por su palrón de once segmentus de doble bebra.

Otra alícuata fue enviada al laboratorio de microbiología de la Facultad de Medicina de la Unuversidad Católica de Chile y analizada dentro de 24 horas por aglutinación de látex (Rotavirus Antigen Latex Tesı, Meridian, U.S.A.) que utiliza partf́culas de lácex recubiertas con antirelavirus policional (cepa SA-II), y por técnica de ELISA (Pathfinder Rolavirus Kallestad, BRD Pasteur Diagnostica. Francia), que utiliza tubos recubiertos con antirntavirus policlonal contra la proteina VP6 (protcína de la cápside internal como anticuerpos de cuptura. Para la identificación del antigeno uxiliza anticuespo monoclonal antirotavirus conjugado con peroxidasa.

Se alnacenaron dos alicuotas a $-20^{\circ} C$, una de las cuales fue utilizada para la prueba ELISA/aglutinación (Test Pack Rotavirus, Abbott Laboratories, IL. U.S.A.), que utibizu partículas ie látex recubiertas con anticuerpo policlonal antiroravirus como fase sólida intnunoabsorbente; la aglutinación de estas partículas sc amplifica mediante el uso de anticuerpos monocionales y policlonales anti- rotavirus conjugados a fosfatasa alcalina. La presencia de RV produce la formación de complejos antígeno-anticuerpo conjugado. los que son retenidos en un filtro $y$ visualizados ante la exposición a un cromógeno. La alícuola restante se utilizó para prueba confirmatocia con anticuerpos bloqueadores por técnica de ELISA que se basa en la disminución del $50 \%$ o más en la densidad óptica después del tratamieato con sutero hiperinmune ${ }^{4}$ il

Las muestras positivas, por cualesquiera de las técnicas ел evaluación, que resultaron discordantes con la prueba confirmatoria fueron procesadas para obstrvacion por inmunoelectronicroscopia: para esta útrima prueba se utilizó anticuerpos policlonales antirotavirus para facilitas la observación del virus (figura).

Los resultados fueron somelidos a prueba de compara cion de proporciones dependientes (prueba de Cochran) ${ }^{21}$

\section{Resultados}

Se incluyeron en el estudio mucstras de deposición obtenidas de 51 pacientes, 21 de los cuales presentaban una diarrca aguda por rotavirus determinado por prueba confirmatoria. Los resultados de cada una de las pruebas diagnosticas evaluadas se muestran en la tabla 1 .

La determinación de sensibilidad, especificidad, valor predictivo positivo y valor predictivo negativo para cada prueba evaluada, usando como estândar la prueba de ELISA confirmatorio, se muestran en la tabla 2. Los métodos de ELISA y RV-Test Pack no presentaron diferencias estadísticamente significativas de sensibilidad con la prueba confimnatoria. La sensibilidad

\section{Tabla l}

Número de muesıras positivas y negativas según método diagnostico

\begin{tabular}{|c|c|c|c|c|c|}
\hline $\begin{array}{l}\text { Resultado } \\
\text { prueba } \\
\text { confirma- } \\
\text { toria }\end{array}$ & $\begin{array}{l}\text { Nómero } \\
\text { muestras }\end{array}$ & Elisa & $\begin{array}{c}\text { Resultados } \\
\text { T. Pack }\end{array}$ & $\begin{array}{l}\text { Otros } \\
\text { métodos } \\
\text { R. Gel }\end{array}$ & Látex \\
\hline \multirow[t]{4}{*}{ Positivo } & 15 & + & + & + & + \\
\hline & 2 & + & + & + & - \\
\hline & 3 & + & + & - & - \\
\hline & 1 & + & - & - & - \\
\hline \multirow[t]{4}{*}{ Negativo } & 25 & - & - & - & - \\
\hline & 3 & + & - & - & - \\
\hline & 1 & - & - & $t^{*}$ & - \\
\hline & 1 & - & + & - & - \\
\hline
\end{tabular}

* Una de las muestras resultó positiva sólo por electroforesis de RNA, corespondiendo probablemente a un parárolavirus. 

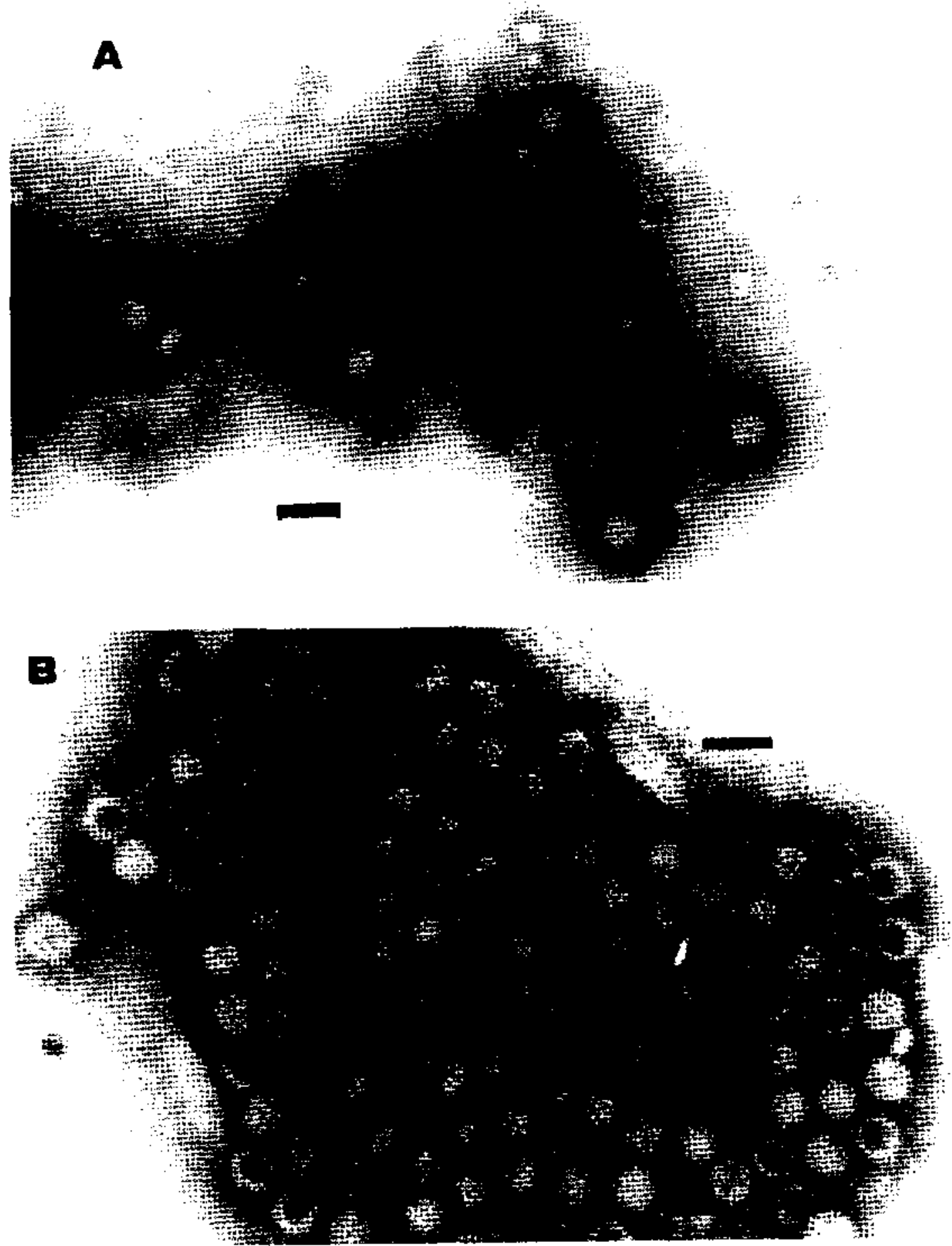

Figura: Observación directa (A) por microstopia elctronica de particulas de rotavirus en deposiciones de un laciutik $4 \%$ diarrea aguda. Inmunoelectromicroscopía (B) de la misma muestra con auticuerpos antirotayirus. Barra: $100 \mathrm{~nm}$. 


\section{Tabla 2}

Sensibilidad, especificidad, valor predictivo positivo (VPP) y valor predictivo negativo (VPN) según método diagnóstico

\begin{tabular}{lcccr}
\hline Método & $\begin{array}{c}\text { Sensi- } \\
\text { bilidad }\end{array}$ & $\begin{array}{c}\text { Especi- } \\
\text { ficidad }\end{array}$ & VPP & VPN \\
\cline { 1 - 5 } Elisa & 100,0 & 90,0 & 87,5 & 100,0 \\
Rotağel & 81,0 & $100.0^{\mu}$ & $100,0^{*}$ & 87,7 \\
Lâtex & 71,4 & 100,0 & 100,0 & 83,0 \\
\hline Test Pack & 95,2 & 96,6 & 95,2 & 97,0
\end{tabular}

* Una de las muestras resultó positiva sólo por electroforesis de RNA, lo que podría corresponder a un pararotavirus (no se confimma por microscopía elecirónica).

de las técnicas Rotagel (81\%) y aglutinación de látex $(71,4 \%)$ fueron significativamente menores ( $\mathrm{p}=0,032$ y $\mathrm{p}=0,0013$ respectivamente). La especificidad de los distintos métodos empleados no mostró diferencias estadísticamente significativas $(p=0,28)$.

No se observaron partículas virales por microscopía electrónica en ninguna de las 5 muestras negativas por ELISA confirmatorio y positivas por algunos de los métodos evaluados. La muestra positiva por electroforesis de ARN fue negativa por microscopía electrónica.

\section{Comentario}

Rotavirus es el principal agente causal de diarrea en lactantes y al menos $3 \%$ de los lactantes infectados requiere hospitalización ${ }^{12}$; al mismo tiempo, RV produce altas tasas de infección intrahospitalaria en servicios de pediatría (12 a $25 \%)^{22,23}$. Debido a que no existe terapia específica, la acción médica esta orientada a prevenir o tratar la deshidratación, que es su principal complicación'12, 13.24. La disponibilidad de métodos rápidos para el diagnóstico de $\mathrm{RV}$ tiene importancia clínica y epidemiológica, permitiendo racionalizar las decisiones de manejo médico y control de infecciones. Considerando la alta incidencia de esta infección y su presentación como cuadro agudo que generalmente motiva consulta en servicios de urgencia. es importante que estos métodos de diagnóstico sean rápidos, seguros, de bajo costo y que no requieran equipo especial ni personal altamente entrenado.
La evaluación presentada aquí fue realizada con un número de muestras que permite determinar diferencias de sensibilidad superiores al $15 \%$ (con un poder estadístico de $80 \%$ para $\mathrm{p}=$ $0,05)$. Los resultados indican que el método ELISA y el RV-Test Pack tienen la mejor sensibilidad (100\% y $95 \%$ respectivamente), ofreciendo la ventaja de tener alta especificidad (90\% y $97 \%$ respectivamente). Las técnicas de Rotagel y aglutinación de látex evaluadas aparecen con una sensibilidad significativamente menor, fallando en detectar la infección en 19 y $29 \%$ de los casos respectivamente. Esta menor sensibilidad no es compensada, como se podría esperar, por una especificidad significativamente mayor. El problema diagnóstico se concentra principalmente en la sensibilidad, la que se ve afectada por diferentes factores, según la técnica empleada. En la prueba de aglutinación de látex se ha descrito interferencia de anticuerpos antirotavirus en deposiciones; estos anticuerpos, de tipo IgA, están presentes tempranamente durante la infección y bloquearían los sitios de reaccion ${ }^{16}$. La electroforesis de ARN viral requiere de un número mayor de partúculas virales para obtener un resultado positi$v^{7,9}\left(10^{7}-10^{11}\right.$ partículas por $\mathrm{ml}$ de deposición versus $10^{5}$ requeridas por ELISA o microscopía electrónica); por otro lado, el ARN viral es lábil y susceptible a degradación por ARN asas normalmente presentes en muestras biologicas. En relación al RV-Test Pack, se ha infortmado que la sensibilidad disminuye cuando la deterninación se realiza con muestras que han sido sometidas a congelación ${ }^{11}$

Estudios publicados previamente, que comparan métodos de diagnóstico para RV, muestran cifras similares de sensibilidad a las informadas aquí ${ }^{7}, 10.16,25$. La prueba de ELISA muestra mayor variabilidad con sensibilidad de $80 \%$ a $100 \% 7,10,16.25$. La sensibilidad de1 RV-Test Pack fluctua entre 95 y $100 \%{ }^{11,25}$, disminuyendo significativamente si se congela la muestra previo a su procesamiento ${ }^{11}$. La sensibilidad de la electroforesis de ARN es significativamente más baja, alcanzando generalmente un $80 \% \%^{6.7}$. Esta técnica puede tener su principal indicación cuando se requiere monitorizar la diseminación del virus en un brote limitado, ya que permite comparar las cepas identificadas en los pacientes mediante el patrón de bandas de su ARN viral $6,24,26-28$. 
Aun cuando las diferencias en la especifidad no fueron significativas, se observaron algunos resultados falsos positivos. En el caso de Rotagel en una muestra se detectó material genómico similar al de rotavirus sin que la muestra fuera positiva por ningun otro método; esto podría representar la presencia de pararotavirus $\mathrm{s}^{22,24}$, sin embargo esto no fue confirmado por microscopja electrónica. La prueba de ELISA presentó 3 falsos positivos (negativos por prueba confirmatoria y por microscopía electrónica) los que probablemente se deben a reacción cruzada con otros antígenos ${ }^{29.31}$; el RV-Test Pack tuvo un falso positivo, de reacción débil, fenómeno que ha sido atribuido a la presencia de anticuerpos de tipo IgM u otras proteínas (proteínas de $S$, aureus. factor reumatoide, etc. $)^{\prime \prime}$.

En la tabla 3 șe presenta información global que podría ser considerada en la toma de decisiones de la técnica a utilizar en cada laboratorio local. Tal como se describe, es difícil conjugar los costos con Ia efíciencia diagnóstica. En base a la sensibilidad, especificidad y valores predictivos, el RV-Test Pack aparece como la prueba de elección para el diagnóstico de RV; adicionalmente es la prueba que tiene un menor tiempo de procesaniento y no requiere de equipamiento adicional: por otto lado su precio es el más elevado de las prucbas analizadas. La prucba de ELISA ticne una eficiencia similar a RVTest Pack en el diagnóstico, pero requiere ma. yores habilidades técnicas y tiempo de procesamiento. El uso de Rotagel tiene limitaciones por su menor sensibilidad y por requerir mayor destreza técnica, pero al mismo tiempo representa la prueba de menor precio, es de fácil disponibilidad y desde el punto de vista clínico

\section{Tabla 3}

Costo, tiempo empleado en la determinación y scnsibilidad de cada una de las pruebas de deteccion de antfgeno para rotavirus evaluadas

\begin{tabular}{lccr}
$\begin{array}{l}\text { Prueba } \\
\text { (US\$) }\end{array}$ & $\begin{array}{c}\text { Costo unitario } \\
\text { (minutos) }\end{array}$ & $\begin{array}{c}\text { Tiempo } \\
\text { realixación } \\
\text { (minutos) }\end{array}$ & $\begin{array}{r}\text { Sensj- } \\
\text { bilidad }\end{array}$ \\
\hline RY-Test Pack & 10,2 & 1,5 & 95,2 \\
Elisa & 6,7 & 90 & 100,0 \\
Aglut, látex & 2.4 & 15 & 71,4 \\
ARN Rotagel & 0,5 & 120 & 81,0 \\
\hline
\end{tabular}

eventualmente permite mantener un seguimiento epidemiológico de las cepas presentes en una comunidad o un hospital.

\section{Resumen}

Las pruebas disponibles para detección de rotavirus ( $R V$ ) presentan diferente eficiencia en el diagnóstico. Se evaluaron aquí cuatro métodos de diagnóstico de RV en deposiciones. Con este objeto se analizaron 51 muestras de niños con diarrea aguda (2l RV positivos) por los métodos de electroforesis de ARN viral (Rotagel, ISP-Chile), aglutinación de látex (Rotavirus Antigen Látex Test, Meridian), ELISA (Pathfinder, Kallestad), e inmunoensayo ligado a aglutinación (RV-Test Pack, Abbott). Todas las muestras fueron corroboradas con un ensayo de ELISA confirmatorio y su resultado fue considerado cl estándar para comparaciones. La sensibilidad y especificidad para ELISA fue 100 y 90\%; RV-Test Pack: 95 y 97\%; electroforesis de ARN 81 y 100\%; aglutinación de látex 71 y $100 \%$. El tiempo de procesamiento por mucs(ra tuvo un rango entre 15 min (RV-Test Pack) a 120 min (Rotagel). El precio de costo por muestra (en US\$) fue: RV-Test Pack 10.2: ELISA. Pathtinder ${ }^{6.7}$; látex 2.4; y Rotagel 0,5. RV-Test Pack aparece como la prueba de elección para el diagnóstico de RV por su rapidez y confiabilidad. sin embargo su precio podría impedir su uso en numerosos centros clínicos. La prueba de ELJSA mostro una eficiencia diagnóstica similar, su precio es más bajo, pero tiene mayores requerimientos técnicns y un tiempo de procesamiento más largo.

(Palabras elave: rotavirus, diagnóstico, métodos rápidos.)

\section{Agradecimientos}

Agradecemos la asistencia técnica de la Sra. Teresa Azócar y Srta. Mercedes Paredes, la colaboración del Sr. Luis Villaroel en el análisis estadístico y la colaboración del personal de los servicios de Pediatría en la toma de muestra y registro de datos.

\section{Referencias}

1. Kapikian AZ, Kim HW, Wyan RG: Reovirus-like agent in stools: association with 10 fantil diarrbea and 
development of serologic test. Science 1974; 185: 1049-1053.

2. Berthiaume L. Alain R. Mc Lukghlin B, ef al.: Rapid detection of human virus in faeces by a simple and routine immune electron microscopy technique. J Gen Virol 1981; 55: 223.227.

3. Flewet TH. F.R.C.P., F.R.C. Path: Electron microscopy in the diagnosis of infectious diarthea. JAVMA $1978 ; 173$ : $538-543$.

4. Rubenstein $A$ and Miller $M$ : Comparison of an enzyme immunoassay with eletron miccoscopic procedures for detecting rotavitus. J Clin Microbiol 1982: 15: 938-944.

5. Trépanier P. Alain R, Mirusan V, ef al.: Comparison of three electron microscopy techniques for detection of human rotaviruses. Microb Immunol 1981: 25: $1019-1024$

6. Espejo RT. Avendaño LF. Muñoz O, et al.: Comparison of human rotaviruses isolared in Mexico City and in Santiago, Chile by electrophoretic migralion of their double - stranded ribanucleic acid genome segments. Infection and Immunity 1988: $30: 342-348$.

7. Avendaño L. Dubinotsky $S$ and James $H$ : Comparison of viral RNA electrophoresis and indirect ELISA methods in the diagnosis of human rotavirus infection. PAHO Bulletis 1984; 18 ; $245-249$.

8. Araya M, Spencer $E$, Brunser O, et al: Escudio comparativo de dos métodos en el diagnóstico de rotavirus en lactantes con diarrea aguda y asintonuáticos. Rev Chil Pediatr 1935; 56: 441-444

9. Espejo R, Romero P, Calderón A. Gonzalez N: Diagnóstico de rotavirus por electroforesis de ARN viral. Bol Med Hosp Infant Mex 1978; 35: 323

10. Chernesky A1, Castriciano S. Mahony J, et al.: Examination of the rotazyme II enzyme immunoassay for the diagnosis of rotavirus gastroenteritis. J Clin Microbiol 1985: $22 ; 46.3-464$.

11. Binoks R, Brown L. and Franklin R: Comparison of new rapid test (Test Pack Rotavirus) with standard enzyme immunoassay and electron microscopy for the detection of rotavirus in symptomatic hospitalized children. J Clin Mictobiol 1989; 27: 775-777.

12. Kapikian $A Z$, and Wyart $R$ : Viral gastrointestinal infections.In Fejgin R.D. and Chery J.D. (eds) Textbook of Pediatric Infectious Diseases 3rd Ed. WB Saunders Co. Philadelphja. 1992: 655-664.

13. Blacklow' $N$. and Greenberg H.: Viral gastroenteritis. New Engl J Med 1991: 325: 252-264.

J4. Browdre J: Viral gastroenteritis and laboratory delection of rotavirus. Am J Medical Techn 1983: 49: 665-668

15. Ward RL. Knowhon DR. Pierce MJ: Efficiency of human rotavirus propagation in cell culture. J Clin Micro 1984; 19: 748-753.
16. Lipson S. and Zelinsky $K$ : Comparison of four latex agglutination (LA) and three enzyme-linked immunosorbent assay (ELISA) for the detection of rotavirus in fecal specimens. An J Clin Pathol 1989; 92: 637-643.

17. Chrystie /L. Tonerdell BM, Banatuala IE: Asymptomatic endemic rotavirus infection in the newbom. Lancet 1978: 1: 1176-1178

18. Banatula JE. Christie $Z$. Flower AJE: Rapid diagnosis of viral infections. Lancel 1975; 2; 79-80

19. Flewett $T$. Ariets $C$. Avendaño $F$, et al.: Comparative evaluation of the WHO and Dakoparts enzyme-linked immunoassay kits for rotavirus detection. WHO Bulletin OMS 1989; 67: 369-374.

20. Gomez. J. Esters M. Matson D, et al.: Serotyping of human rotavirus in Argentina by ELISA with monoclonal antibodies. Arch Virol 1990; I12: 249-259.

21. Conover W: Practical nonparametric statistics. $2 d$ Ed. John Wiley and sons, New York. 1980: 199-206.

22. Burraza $P_{1}$ Avendaño $L$, Spencer $E$, et al.: Infección intrahospitalaria por rotavirus en laclantes. Santiago. Chife. Bol Sanit Panam 1986; 101: 328-337.

23. Cone $R$. Mohan $K$, Thouless $M$. et at: Nosocomial transmission of rotavirus infection. Pediatr Infect Dis J $1988 ; 7: 103-108$

24. Cukor $G$ and Bhacklow N. Husnan viral gastroenieritis. Microbiological Reviews 19B4; 48: 157-179.

25. Murlen'jcz B. Spiewak M. Lampinen J: Evaluation of ABBOT Test Pack Rotavins with clinical specimens. J Clin Microbiol 1988: 36: 2456-2458.

26. Corteria $M$, Vial $P$, Nataro J: Diagnóstico de la diarrea aguda. Boletín Escuela de Medicina Pontificia Universidad Catolica de Chile 1988: 18: 38.44

27. Dolan K. Thist EM. Horton-Slight P. et al.: Epidemiology of rotavirus electropherotypes determined by a simplified diagnostic technique with RNA analysis. J Clin Microbiol 1985; 21:753 - 758 .

28. Vial PA. Kotloff $K$. Losonshy $G$ : Molecular epidemiology of rotavirus infection in a newbors convalescent room. J Infect Dis 1988: 157: 668 - 673.

29. Brandt $C$. Wha $H$, Rodriquez $W$, et al.: Comperison of direct electron microscopy, immune electron microscapy and rotavirus enzyme linked immunoassay for detection of gastroenteritus viruses in children. I Clin Microbiol 1981; 13: 976-981.

30. Hermonn $J$, Blacklow $N$, Perron D. et al: Enzyme immunoassay with monoclonal antibodies for the detection of rotavirus in stool specimens. J Infect Dis $1985 ; 152: 830-832$.

31. Brandt $C$, Parrof $R H$, Kim $H V$, et al: Diarthea Viruses: detection, specific identification and epider miology in L.M. de la Maza and Peterson (ed) Me. dical Virology III. Elsevier Science Publishing Co., New York 1984: 35-65. 